\title{
Functional genomics touches base
}

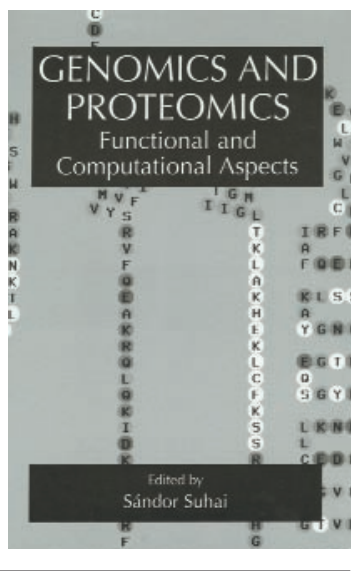

\author{
Genomics and Proteomics: Functional and Computational Aspects \\ edited by Sandor Suhai
}

Kluwer Academic/Plenum Publishers · June 2000

Hardback £94/\$135

Philippe Vaglio and Marc Vidal

A stated in one of the chapters of this book, "genomics does not end when all base pairs of DNA have been sequenced". Large-scale sequencing projects have triggered a giant step for biological sciences. But in the aftermath of the working draft for the human genome, biologists are now left with an even greater number of questions. Undoubtedly, they will have to use new approaches to address them. In "Genomics and Proteomics", the reader will find a description of some of the new challenges but also the opportunities brought about by the post-genomic era. Challenges include finding the correct structure of genes at the DNA level or determining the three-dimensional structure of a large numbers of proteins and elucidating their functions. The opportunities described here include measuring transcript or protein product levels for all genes and drawing a nearly complete picture of a chromosome's architecture.

The book gives a good sample of the emerging fields of functional genomics and proteomics. An increasing number of biologists have to face the field of bio-informatics which is now becoming an integrated part of the post-genomic era. Eight chapters introduce the reader to many of the different bio-informatics approaches currently being developed. These include sequence assembly, gene predictions within the genomic sequence, sequence homology searches, 3D structure predictions, and development of tools and databases for the analysis and visualization of data by biologists. Particularly, one chapter nicely describes the requirements for the launching of large-scale proteome-wide "structural genomics" projects. One of the main qualities of this book it that most of the chapters attempt to bring together the needs of conventional biology with the new possibilities offered by the functional genomics and proteomics fields. In that sense, many "non-genomicists" will find it interesting. We nevertheless saw two weak points in this publication. First, many of the chapters describe large sets of very detailed data sets, which would not be compatible with a textbook setting. College students might get relatively confused. Second, specialists in the field of genomics might be a little frustrated by the fact that some of the information is already getting old. In an area of biology that is probably moving faster than any other (who would have thought 2 years ago that the sequencing of the human genome would be complete by February 2000?) books like these can become rapidly out of date.

Philippe Vaglio and Marc Vidal are at the

Dana-Farber Cancer Institute, Department of Genetics, Harvard Medical School,

Boston, USA

email:Marc_Vidal@dfci.harvard.edu

\section{Proteomics}

edited by S R Pennington and M J Dunn

BIOS Scientific Publishers Ltd

September 2000, £29.99/\$44.95

\section{Sarah Greaves}

$\Lambda$ s we all know, the human genome has recently been sequenced (see Nature 409, 745-964 and Science 291, 1153-1369), and so now the attention of researchers everywhere must focus on the analysis of the proteome. Proteomics is a relatively new field of research that encompasses all recent developments in the analytical methods used to study protein characterisation. This new book is split into 15 chapters, each written by experts in the field from both academia and industry, to introduce the field of proteomics to a wide-ranging audience. The chapters cover an elementary introduction to the field by explaining the link between genomics and proteomics, and hence its importance. Early chapters in the book go on to detail the techniques used for proteome analysis: measurement of mRNA expression, protein detection, image analysis of 2D gels and the use of mass spectrometry to identify proteins and phosphorylation sites. These chapters explain the details behind the methods but readers will have to look elsewhere for the exact protocols needed to perform the complex procedures. However, these chapters are written in an easy to understand manner and will appeal to both undergraduates and new researchers in the field. Later chapters go on to discuss the implications of proteomics in an academic research environment and as a potential tool for plant genetics and breeding. These chapters also discuss the future for the proteomics field and its development within scientific research.

Although the book is pitched at final year undergraduates, all readers with a general interest in this fast expanding field will benefit from having a copy on their shelves.

\section{Other proteomic and genomics books}

Functional Genomics

edited by S. Hunt and F. Livesey

Oxford University Press, £29.95/\$49.95

Comparative Genomics

edited by David Sankoff and

Jospeh H. Nadeau

Kluwer University Press, £49/\$79

Proteome Research: New Frontiers in Functional Genomics

edited by M. R. Wilkins, K. L. Williams,

R.D. Appel and D. F. Hochstrasser

Springer-Verlag, £26/\$48.95

Genomics

by John E. Antonopoulus

Xlibris Corporation, £20/\$25

Comparitive Genomics

by Melody Clark

Kluwer Academic Press, £77/\$110

Deciphering the Book of Life by Michael Q. Zhang

World Scientific Publishing Co., £46/\$67 